\title{
An Argument for the Ontological Innocence of Mereology
}

\author{
Rohan French ${ }^{1}$
}

Received: 17 September 2013/Accepted: 22 August 2015/Published online: 23 September 2015

(C) The Author(s) 2015. This article is published with open access at Springerlink.com

\begin{abstract}
In Parts of Classes David Lewis argued that mereology is 'ontologically innocent', mereological notions not incurring additional ontological commitments. Unfortunately, though, Lewis's argument for this is not fully spelled out. Here we use some formal results concerning translations between formal languages to argue for the ontological innocence of mereology directly.
\end{abstract}

\section{Introduction}

On the face of it a theoretical commitment to unrestricted mereological composition-that any collection of objects, no matter how disparate, has a mereological fusion-has a steep ontological cost. For example, suppose that there is a cat, Tom, and a mouse, Jerry. By unrestricted mereological composition we appear to be committed to the existence of a further entity - their fusion-distinct from either of them. On the face of it, then, it appears that unrestricted mereological composition commits us to the existence of additional objects. In response to this kind of argument Lewis says the following.

To be sure, if we accept mereology, we are committed to the existence of all manner of mereological fusions. But given a prior commitment to cats, say, a commitment to cat-fusions is not a further commitment. The fusion is nothing over and above the cats that compose it. It just is them. They just are it. Take them together or take them separately, the cats are the same portion of Reality either way (Lewis 1991, p. 81).

Rohan French

rohan.french@gmail.com

1 Faculty of Philosophy, University of Groningen, Oude Boteringestraat 52, 9712 GL Groningen, The Netherlands 
So, according to Lewis, mereology does not commit us to the existence of 'additional' entities such as the fusion of Tom and Jerry: it is, in some sense, "ontologically innocent". Lewis provides some support for this view by pointing out certain similarities between mereological relations (such as parthood and fusion) and identity. For example, if you are committed to the existence of an object $a$, and $a$ is identical to $b$ then you are not burdened with any additional ontological commitment to $b-b$ would seem to come as an ontological 'free lunch' in the presence of a prior commitment to $a$. In fact, Lewis claims that mereological relations are so closely related to identity that this ontological innocence transfers over to the mereological relations. ${ }^{1}$

Lewis's argument for the ontological innocence of mereology is an argument by analogy. First we note that identity is "ontologically innocent". Secondly we note that there are a number of similarities between mereological relations and identity, such as: unrestricted applicability, uniqueness of applicability, ease of description, and the co-location of things which they relate. From this we are then meant to conclude that the mereological relations are also "ontologically innocent". This argument has one of the usual flaws which arguments by analogy have, namely that we are given no reason for thinking that similarity in the listed respects is sufficient for the mereological relations to be ontologically innocent [on which see section 3 of Yi (1999)]. ${ }^{2}$ The argument is made even more vexing in that one of the ways in which Lewis claims identity and the mereological relations are analogous is precisely the one under dispute: namely, ontological innocence! What we will do here is argue that Lewis is indeed correct to think that mereology is ontologically innocent, using formal methods to provide a more direct argument. Throughout when we talk of mereology we will mean classical extensional mereology, the formal theory which combines Lewis's commitment to universal mereological composition (that any objects have a fusion), with the extensionality of parthood (objects with the same parts are identical). Both of these features of classical extensional mereology will be needed in our argument.

There is a pressing question which we need to settle first, though. Namely, what exactly is it that we are claiming about mereology—what does Lewis really mean (or could he mean) by "ontologically innocent"?

\footnotetext{
${ }^{1}$ It is somewhat unfortunate that Lewis decided to refer to this thesis as Composition as Identity, given that he explicitly denies that he is adopting the thesis as it is commonly understood (as in, e.g. Baxter 1988). What Lewis really means by 'composition as identity' is that, in some sense, mereological relations are instances of 'identity in the broadest sense', the limiting case of which is ordinary (numerical) identity (Lewis 1991, p. 84f). For the state of the art concerning the thesis of composition as identity the reader should consult Cotnoir and Baxter (2014).

2 One could sufficiently strengthen the analogy between mereological relations and identity by giving a generalisation of identity in plural quantificational terms which has the appropriate formal characteristics and collapses to identity between objects under certain circumstances. Lewis explicitly points out that he can see no way to do this, though (cf. Lewis 1991, p. 87). Further difficulties for this line of argument are discussed in $\$ 3$ of Sider (2007).
} 


\section{Ontological Innocence?}

Lewis is not explicit about what exactly it takes for a theory to be ontologically innocent. We are told that plural quantification is ontologically innocent, because it just gives us a new, primitively plural, way of talking about the first-order domain of quantification rather than committing us to the existence of exotic plural entities, classes or sets. By contrast set theory is not ontologically innocent: given a commitment to an object $a$ we are suddenly committed to a multitude of objects distinct from $a$, namely the various sets we can construct out of $a$, such as $\{a\}$, $\{\{a\}\}$ etc. ${ }^{3}$ Mereology, though, is meant to be different.

Mereology is innocent in a different way: we have many things, we do mention one thing that is the many taken together, but this one thing is nothing different from the many (Lewis 1991, p. 87).

So mereology is different as far as ontological innocence is concerned from both plural quantification - as there is something (the fusion) which is the many taken together-and also set theory - as this something is not distinct from the many out of which it is composed. Now, if we interpret this remark strictly and literally, what Lewis is saying here cannot be true. After all, as Lewis himself notes in the paragraph previous to that where the above quote appears, the number of lemons in the fruit bowl on the table as I write this is four, but the number of their fusion is one. So there is at least some respect in which the fusion and the fused differ. How, then, should we understand this claim that mereology is "ontologically innocent"?

One way of attempting to cash out this idea is as follows. Let us, following Hodes (1990), draw a distinction between what a sentence $S$ says exists (its thin commitments), and what is required for the sentence $S$ to be true, the facts which are relevant to settling $S$ 's truth-value (its thick commitments). One way of cashing out what it means for a theory to be ontologically innocent is the following: ${ }^{4}$

INNOCENCE: a theory $T$ is ontologically innocent regarding the existence of $X \mathrm{~s}$ iff $X$ s are amongst $T$ 's thin commitments (i.e. it asserts that $X$ s exist) but not amongst its thick commitments (i.e. the truth of the theory does not require the existence of $X \mathrm{~s})$.

For example (approximately speaking) in the case where $T$ is mereology, to show that mereology is ontologically innocent regarding the existence of mereologically complex objects (our $X \mathrm{~s}$ in this situation) one needs to show that mereologically complex objects are amongst the thin commitments of mereology, but not among its thick commitments. ${ }^{5}$ This characterisation is approximate mostly due to being slightly too strong, and we'll qualify this slightly in Sect. 8.

\footnotetext{
${ }^{3}$ Lewis (1991) is an attempt to found set-theory on mereology and plural logic-what Lewis calls 'Megethology'. Using the resources of Megethology Lewis argues that set-theory can be reduced to the theory of singletons. We leave it to the interested reader to examine how well Lewis's megethological manoeuvres sit with our argument for the ontological innocence of mereology below.

4 According to the following definition, theories which are ontologically innocent regarding the existence of $X$ s would seem to count $X$ s as thin existents in the sense of Linnebo (2012).

5 A referee points out that this definition of ontological innocence may be inadequate because classical extensional mereology is not a conservative extension of theories of the world which are able to state
} 
In Hodes (1990) the distinction between thick and thin commitments is understood as being a distinction pertaining to the syntactic and semantic forms of sentences under discussion in a particular domain. This is, of course, not the only way of spelling out something like this distinction, and it will not be ours here. Suppose that we are dealign with a theory $T$ which is 'ontologically transparent'so it is obvious what its ontological commitments appear to be. Then we will say that $T$ has a thin commitment to $X \mathrm{~s}$ iff there is a truth (and falsity) preserving translation from $T$ to some other ontologically transparent theory $T^{\prime}$ which is not committed to $X$ s. We probably also want to place other requirements on the theories $T$ and $T^{\prime}$ (for example they should both have plausible metaphysical/ideological commitments, and perhaps both be able to adequately represent reality). ${ }^{6}$ Making use of this distinction allows us to make some sense of what precisely Lewis is claiming of mereological fusions, especially if we think of the thin commitments of a sentence as supervening on its thick commitments. It is clear in this case how the fusions and the fused might then be construed as being the "same portion of Reality', and in what way fusions are not distinct from the fused. Our proposal will ultimately be similar to one informally presented by Ted Sider over the course of trying to determine exactly what talk of fusions being "nothing over and above their parts' might mean.

[O]ne might construe "the whole is nothing over and above the parts" as implying that the features of the whole are in some sense nothing over and above the features of the parts. ... Any irreducibly macroscopic features can be pinned on relations between the parts, and therefore do not essentially involve the whole to the exclusion of the parts (Sider 2007, p. 73).

The important question, given the above line of thinking, is how exactly we are supposed to spell this out.

\footnotetext{
Footnote 5 continued

constraints on the number of objects which exist. For example, if $T$ is a theory of the world which claims that there are only two objects then this theory will not be conservatively extended by the theory $M$ of classical extensional mereology in the sense that there will be sentences solely in the language of $T$ (such as the claim that there are more than two objects) which are true in $T+M$ but not in $T$ - this being essentially the point pressed in Comesaña (2008). I think there are two issues with this line of argument against the claim the mereology is ontologically innocent. Firstly, counting objects in the presence of mereological principles requires some amount of care: for example, we ought to avoid (significant) overlap between the objects counted in order to avoid double counting. Secondly, and more importantly, the notion of innocence tracked by conservative extension is far stronger than the notion we have in mind here. If the addition of mereological principles conservatively extended a theory of the world then, given that the theory of the world can talk about how many objects there are in its original language, this would suggest that mereology was ontologically innocent by not bringing with it any ontological commitments at all, this is essentially the manner in which Lewis claims that plural logic is ontologically innocent. What is claimed here, though, is that mereology brings with it a commitment to mereologically complex objects, but that this commitment is 'thin' or 'lightweight'.

${ }^{6}$ This is similar to the proposal made in Williams (2012) concerning 'requirements on reality'. There 'thick commitments' - Williams' 'reality requirements' - are the objects which your metasemantic theory commits you to. As this reference to metasemantics makes clear, Williams is concerned with an interesting and sophisticated kind of paraphrase strategy, which the present suggestion should be distinguished from. Paraphrase strategies, in general, are arguments for not being committed to things, not for a commitment to them being innocent.
} 


\section{Our Approach in Outline}

Our approach will be to show that mereology is ontologically innocent using formal models. Speaking loosely, we will show that everything which can be said in the language of classical extensional mereology can be said in a language free of mereological primitives, and in particular free of quantification over mereological fusions. To do this we will provide a translation function which maps sentences from our mereological language to sentences of our non-mereological language. Thinking of the non-mereological language as being one in which the thick commitments of our mereological language are best expressed, this will show how claims that 'there is a fusion of Tom and Jerry' only commit us (in the thick sense) to Tom and Jerry-and thus by INNOCENCE that mereology is ontologically innocent.

As with any formal modelling it will be helpful to make a few simplifying assumptions in order to make our formal work more tractable. In what follows we will make the following assumptions:

- Worldbound: we will only be concerned with the actual world (or any single world) and its denizens.

- No Gunk: we will also assume that there is no atomless gunk in the world under consideration. That is, every object is composed out of mereological atoms.

- Limitation of Size: we will assume that there are only countably many mereological atoms.

- Anonomism: we will assume that names and other singular terms are not necessary for a complete description of the world.

The first assumption serves to allow us to bow out of any debates concerning the essentiality of mereological composition. This assumption also justifies our concerning ourselves with purely extensional languages. The third assumption is important as it allows us to consider slightly more tractable languages in our translation, as we will discuss in Sect. 4.2.2. The fourth assumption plays a more subtle and indirect role in what follows. This thesis is argued for explicitly (under this name) in Karmo (1985), and is a feature of languages which are intended to be 'purely qualitative', such as those used in arguments for anti-haecceitism in modal metaphysics [such as that in Lewis (1986, p. 221)]. Its purpose here is to allow us to restrict attention to languages which do not contain individual constants or singular terms. These three assumptions (Worldbound, Limitation of Size, and Anonomism) are made purely for the sake of convenience, allowing us to simplify our formal modelling. How this is so is clarified in our conclusion. The situation concerning No Gunk is slightly more delicate, though.

No Gunk is probably both the most important, and also most contentious of the above assumptions, especially given arguments that gunky worlds are possible such as in Sider (1993). We are not alone in making this assumption (van Inwagen 1990), which Sider is responding to, makes it also), but the issue is rather delicate. For the moment we will adopt it nonetheless because of its technical utility. We will return at the end of the paper, in Sects. 8 and 9, to examine the extent to which our arguments really do rely on these assumptions. 


\section{Two Formal Languages}

Before talking about our translation we will first need to introduce the two formal languages which will be taking centre stage in the result to follow: the first-order language of mereology, and our 'flexible infinitary language'.

\subsection{Mereology}

Let $L_{m}$ be a first-order language with a distinguished binary predicate ' $\square$ ' for parthood. The quantifiers of this language range over, not just the mereological atoms, but also their fusions. Here we are taking the relation of parthood as our mereological primitive, although nothing substantial hangs on this choice, as all of the other mereological relations such as 'overlap', 'proper-parthood' can be defined directly in terms of parthood in the language we are considering - to take a simple example, $a$ is a proper part of $b$ if and only if $a$ and $b$ are distinct and $a$ is a part of $b .^{7}$

Models for $L_{m}$ are models for first-order logic with an added interpretation for the parthood relation, and will be of the form of the form $\mathcal{M}=\langle D, \sqsubseteq, I\rangle$. No issues will arise out of our double usage of $\sqsubseteq$ for both the parthood relation in our object language and its interpretation in our models. The distinctive clause for truth in a model $\mathcal{M}$ relative to a variable assignment $\mu$ being:

$$
\mathcal{M} \models x \sqsubseteq y[\mu] \Longleftrightarrow \mu(x) \sqsubseteq \mu(y) .
$$

In particular we will be concerned with models for what is usually called 'atomistic classical extensional mereology': the theory of the part-whole relation where everything is composed out of mereological atoms, where every collection of objects composes some object, and where mereologically complex objects with the same proper parts (and thus the same atomic proper parts) are identical. Thus the structures $\langle D, \sqsubseteq\rangle$ which we will be concerned with are all isomorphic to complete atomic boolean algebras with their least element removed.

\subsection{Infinitary Flexible First-Order Logic}

The other language which we will be using here is slightly more exotic, deviating from that of standard first-order logic in two main respects. Firstly, it has what are referred to in Taylor and Hazen (1992) as flexible predicates. Secondly, it is an

\footnotetext{
7 Taking mereological composition as our primitive would complicate things slightly, requiring us to do some preprocessing of formulas. The standard treatment of mereological composition, which can be seen in Simons (1987), is where we have a primitive variable-binding term-forming operator " $\sigma x F x$ " for the sum of the $F$ s. To treat this we first remove all occurrences of $\sigma x F x$ in favour of $l$-terms by replacing $\sigma x F x$ by

$\iota x \forall y(x \circ y \equiv \exists z(F z \wedge z \circ y))$,

where " $x \circ y$ " is overlap, which can be defined in terms of " $\square$ " (Simons 1987, p. 37). We then remove all such $l$-terms using Russell's contextual elimination of definite descriptions, leaving us with a sentence in $L_{m}$ which we can then apply our translation to. Kit Fine's characterisation of mereology (as being the theory of sum operators which satisfy his conditions CLAP) in Fine (2010) can be dealt with similarly by treating a term $\Sigma\left(x_{1}, \ldots, x_{n}\right)$ just as the above pre-processing tells you to treat $\sigma x\left(x=x_{1} \vee \cdots \vee x=x_{n}\right)$.
} 
infinitary language: allowing infinite conjunctions, disjunctions and blocks of quantifiers of the same kind. We will discuss each of these aspects of this language in turn.

\subsubsection{Flexible Predicates}

In standard first-order logic, predicates are treated as having a fixed arity $n$, combining with $n$ terms to form a formula. One can generalise this situation by allowing predicates to take any number of terms and produce a formula. Doing this gives us multigrade predicates. Multigrade predicates are only really a generalisation of unary predicates, not allowing us to adequately express relations between varying numbers of objects. Flexible predicates are a natural generalisation of both standard ('Fregean') predicates, and multigrade predicates. Flexible predicates, like standard Fregean predicates, have a fixed number of argument places; but each of those argument places may be occupied by a variable number of terms. In more formal terms we associate with each $n$-place flexible predicate $R$ a type which is an $n$-element sequence of pairs (called 'place limitations') telling us the minimum and maximum number of terms which can be inserted into that argument position to form a formula. So, for example, the flexible unary predicate '...live together' requires at least two terms to form a formula-so ' $a$ live together' is not wellformed, while ' $a, b$ live together' is.

The semantics of flexible predication is as one would expect. A flexible predicate is true of some objects inserted into its various argument places in some particular order (respecting that argument places place limitation) whenever those objects in that order in those argument places are in the extension of the predicate. So, for example, let us consider the predicate Between which we will take to be of type $\langle[1, \omega],[1,1],[1,1]\rangle$-allowing any number of things to be between its second and third arguments. In this case $\operatorname{Between}(2,4,6 ; 1 ; 7)$ will be true, as 2,4 , and 6 are between 1 and 7 .

Ultimately the use of flexible predicates here is a convenience which we could dispense with by replacing each flexible predicate with a set of Fregean predicates [for more details here see Taylor and Hazen (1992, p. 385)]. What the flexible predicate do, though, is allow us to state things in a rather perspicuous manner, without making any great presumptions about the nature or commitments of other pieces of vocabulary. ${ }^{8}$

\footnotetext{
${ }^{8}$ One might wonder why we are not using plural predicates and relations instead of flexible ones. Our reason for this is simple: we take it that it would be more perspicuous to show that mereology is ontologically innocent while presuming the ontological innocence of the minimum amount of other primitives. One might reasonably expect that plural quantification is ontologically innocent (and indeed I think that methods similar to those used below can be used to demonstrate as much), but it is hoped that the innocence of mereology does not rest of the innocence of plural quantification. As flexible predicates can simply be replaced by appropriate Fregean predicates and relations, they would seem to be as innocent (ontologically speaking) as Fregean predicates themselves.
} 


\subsubsection{Infinitary Devices}

Given a first-order language $L$, the language $L_{\kappa \lambda}$ (where $\kappa$ and $\lambda$ are infinite cardinals such that $\kappa \leq \lambda$ ) extends $L$ by allowing conjunctions and disjunctions of cardinality $<\kappa$, and uniform blocks of quantifiers of length $<\lambda$. More formally $L_{\kappa \lambda}$ results from $L$ by adding sufficient variables to $L$ so that the cardinality of the set of variables is at least $\kappa \cdot{ }^{9}$ In particular we will be concerned with the language $L_{\omega_{1} \omega_{1}}^{f}$ where $L^{f}$ is the first-order language with flexible predicates described above. That is, we are going to be concerned with the flexible predicate language where we are allowed conjunctions, disjunctions and blocks of quantifiers of any size less than the first uncountable ordinal. Models for $L_{\omega_{1} \omega_{1}}^{f}$ are models $\langle D, I\rangle$ for the flexible predicate logic described above. We will use the same symbol to talk about infinite and finite conjunctions and disjunctions. So, writing $\mathcal{M} \Vdash \varphi[\mu]$ for truth in $\mathcal{M}$ on $\mu$ of formulas $\varphi$ from $L_{\omega_{1} \omega_{1}}^{f}$ we have clauses like the following:

$$
\begin{aligned}
& \mathcal{M} \Vdash \exists x_{1} \exists x_{2} \cdots \varphi[\mu] \quad \Longleftrightarrow \quad \text { there is an assignment } \mu^{\prime} \text { differing at most on } \\
& x_{1}, x_{2}, \ldots \text { from } \mu \text { s.t. } \mathcal{M} \Vdash \varphi\left[\mu^{\prime}\right] \text {. } \\
& \mathcal{M} \Vdash \varphi_{1} \vee \varphi_{2} \vee \cdots[\mu] \quad \Longleftrightarrow \quad \mathcal{M} \Vdash \varphi_{1}[\mu] \text { or } \mathcal{M} \Vdash \varphi_{2}[\mu] \text { or } \cdots
\end{aligned}
$$

These infinitary devices are plausible candidates for being primitives which are required in order to give a complete theory of the world. ${ }^{10}$ The idea here is that if we want to produce a theory $T$ which is a complete theory of the world ('Reality' as Lewis says above) then one of the things which that theory would have to do is place constraints upon the number of things which exist in the world. So it seems that this theory $T$ would have to entail that 'there are at least $n$ things', where $n$ is the number of entities which exist in Reality. Now if there are denumerably many entities (as we are supposing here that there are) then this would mean that $T$ would have to entail the following sentence:

$$
\exists x_{1} \cdots \exists x_{n} \cdots\left(x_{1} \neq x_{2} \wedge \cdots \wedge x_{1} \neq x_{n} \wedge \cdots \wedge x_{n} \neq x_{n+1} \wedge \cdots\right)
$$

This is at least suggestive of the fact that a language like $L_{\omega_{1} \omega_{1}}$ is needed for giving a complete description of the world. Given, then, that the project of showing that mereology is ontologically innocent just is the project of showing that mereologically complex objects are not needed in a complete theory of Reality (and thus plausibly not part of Reality itself) the use of these primitives seems perfectly warranted.

\section{The Translation}

What we will do here is extend an idea proposed in Morton (1975) of translating claims of the form ' $x$ is a cat' (for example) as ' $x_{1}, x_{2} \ldots$ make up a cat' where the $x_{i}$ are

\footnotetext{
9 For further information on Infinitary Logic the interested reader should consult Bell (2012) and the references therein.

${ }^{10}$ Kit Fine gives similar motivating remarks for the use of infinitary modal languages in attempting to simulate possibilist discourse in Fine (1977, p. 147).
} 
all mereological atoms. Morton does this by translating the language of mereology into a language with multigrade predicates. One problem with Morton's translation, though, is that it is unclear how we are to translate predicates of arity $>1$, as Morton only has primitive unary multigrade predicates, and his method of constructing multigrade predicates out of standard predicates, the predicate-functor ${ }^{*}$, gives the wrong answers. ${ }^{11}$ For example according to Morton (1975), 'John is married to Kate' comes out as equivalent to 'every atomic part of John is married to some atomic part of Kate', or perhaps 'every atomic part of John is married* to some atomic part of Kate'. It's not clear that this kind of distributive reading of relations between mereologically complex individuals is always going to work-even if we allow a change in meaning of the predicate in the non-mereological language (so we have 'married"' instead of 'married').

On our approach a sentence like 'John is married to Kate' would be treated as 'the atomic parts of John stand in the flexible relation married $f$ to the atomic parts of Kate'. ${ }^{12}$ It may be helpful to read many of the predicates $P^{f}(\ldots)$ as “... are arranged $P$-wise", and flexible relations $R^{f}(\cdots ; \star \star \star)$ as “ $\cdots$ are related $R$-ly to $\star \star \star$ ". Thus 'John is married to Kate' becomes 'the atomic parts of John are related marriagely to the atomic parts of Kate". It is worth stressing at this point that the relation married $f$ has two argument places (the place limitation for both argument places being $\langle 1, \omega\rangle)$. This means, for example, that the atomic parts of John can stand in this relation to the atomic parts of Kate, without the atomic parts of the upper halves of John and Kate standing in the relation to their lower halves, as would happen if we treated married $f$ as a unary multigrade predicate in Morton's system. ${ }^{13}$ A perhaps more promising line, and one which is definitely more in tune with the intended application of our translation procedure, is to think of flexible predicates and relations as picking out a special kind of (most likely non-natural) property. So, for example, we might think of the flexible predicate chair $^{f}$ as picking out the flexible property the parts $x_{1}, x_{2}, x_{3}, \ldots$ of an object standing in which is sufficient for them to constitute a chair. This would make, in essence, our flexible predicates be the linguistic correlates of the relations mentioned in the characterisation of how to understand the idea of the features of a whole being nothing over and above the features of its parts discussed in Sider (2007, p. 74), and mentioned in the quote at the end of Sect. 2.

In addition to its clear debt to Morton's translation, our translation also draws quite direct inspiration from the translation mentioned by Gödel in his discussion of Russell's 'No-Class' theory in Gödel (1944), as well as similar translations discussed in Fine (1977) and Quine (1947). Our translation $\tau$ from the language $L_{m}$ to the language $L_{\omega_{1} \omega_{1}}^{f}$ discussed above is as follows.

\footnotetext{
11 Morton's translation also makes essential use of quantifiers over sequences of objects, making it inappropriate for our task of showing that mereology is ontologically innocent.

12 Throughout we adopt a general convention of writing the flexible predicate associated with a given predicate in the mereological language by superscripting it with an ${ }^{f}$. So, for example, the flexible binary relation associated with a binary relation $R$ from the mereological language will be written as $R^{f}$.

13 I would like to thank an anonymous referee for pushing me to clarify this point.
} 


$$
\begin{aligned}
\tau\left(R v_{1} \ldots v_{n}\right)= & R^{f}\left(x_{1}^{v_{1}}, x_{2}^{v_{1}}, \ldots ; \ldots ; x_{1}^{v_{n}}, x_{2}^{v_{n}}, \ldots\right) . \\
\tau(\forall v \varphi)= & \forall x_{1}^{v} \forall x_{2}^{v} \cdots \tau(\varphi) . \\
\tau(v \sqsubseteq u)= & \forall x\left(\left(x=x_{1}^{v} \vee x=x_{2}^{v} \vee \cdots\right) \rightarrow\right. \\
& \left.\exists y\left(\left(y=x_{1}^{u} \vee y=x_{2}^{u} \vee \cdots\right) \wedge x=y\right)\right) . \\
\tau(v=u)= & \tau(u \sqsubseteq v) \wedge \tau(v \sqsubseteq u) .
\end{aligned}
$$

Unpacking the above translation, we translate relations between individuals in the mereological theory in terms of flexible relations amongst their atoms, universal quantification in the mereological language is treated as universal quantification over all possible collections of atoms, and ' $v$ is a part of $u$ ' is translated as 'every atom of $v$ is an atom of $u$ '. So, for example, the formalisation of a sentence like "something is a cat" — $\exists x \operatorname{Cat}(x)$ — would become $\exists x_{1} \exists x_{2} \ldots \operatorname{Cat}^{f}\left(x_{1}, x_{2}, \ldots\right)$ (e.g. "some atoms are arranged cat-wise" or "some atoms stand in the flexible catconstituting relation"). Similarly, a sentence like "every cat has a part which is a tail"— $\forall x(\operatorname{Cat}(x) \rightarrow \exists y(y \sqsubseteq x \wedge \operatorname{Tail}(y)))$ —would become:

$$
\begin{aligned}
& \forall x_{1} \forall x_{2} \ldots\left[\operatorname { C a t } ^ { f } ( x _ { 1 } , x _ { 2 } , \ldots ) \rightarrow \exists y _ { 1 } \exists y _ { 2 } \ldots \left(\forall u \left(\left(\bigvee_{i<\omega} u=y_{i}\right) \rightarrow\right.\right.\right. \\
& \left.\left.\left.\quad \exists v\left(\left(\bigvee_{j<\omega} v=x_{j}\right) \wedge u=v\right)\right) \wedge \operatorname{Tail}^{f}\left(y_{1}, y_{2}, \ldots\right)\right)\right]
\end{aligned}
$$

That is to say, "for any atoms which stand in the flexible cat-constituting relation, some of those atoms also stand in the flexible tail-constituting relation".

Using our translation we can show that everything which can be said in the mereological language $L_{m}$ can be said in the non-mereological language $L_{\omega_{1} \omega_{1}}^{f}$. More formally, given a model $\mathcal{M}$ of the mereological language which contains at most denumerably many atoms, we define a unique model $\mathcal{M}^{-}$of our nonmereological language whose domain consists solely of mereological atoms, with a flexible predicate $R^{f}$ being true of the atomic parts of an object when, according to $\mathcal{M}$, their fusion stands in the relation $R$. We can think of this model as representing the underlying 'facts' in virtue of which the statements of the mereological language are true. We can see our translation, then, as mapping sentences $\varphi$ of the mereological language, to statements $\tau(\varphi)$ in our language which does not contain mereological primitives (making commitments to mereologically complex objects thin ones). Formally speaking, this means that whenever $\varphi$ is true in $\mathcal{M}$ then $\tau(\varphi)$ is true in $\mathcal{M}^{-}$. So, in particular, if $\mathcal{M}$ and $\mathcal{M}^{-}$are intended models of Reality, our translation will be truth preserving tout court (rather than simply 'truth in a model' preserving). We leave the full formal details of this to the Appendix. 


\section{Comparison with Other Translations}

To give readers a clearer idea of how our translation works let us consider an example treated in van Inwagen (1990, p. 109). There, van Inwagen paraphrases the sentence 'Some chairs are heavier than some tables', as: ${ }^{14}$

There are $x x \mathrm{~s}$ that are arranged chair wise and there are yys that are arranged table wise and the $x x$ s are heavier than the yys.

Our translation essentially applies a modified form of van Inwagen's procedure with plural quantification replaced by infinitary quantification over mereological atoms, and van Inwagen's plural predicates replaced by appropriate flexible predicates. So, for example, the above sentence would be translated as

There are atoms $x_{1}, x_{2}, \ldots$ such that $\operatorname{chair}^{f}\left(x_{1}, x_{2}, \ldots\right)$ and there are atoms $y_{1}, y_{2}, \ldots$ such that table $^{f}\left(y_{1}, y_{2}, \ldots\right)$ and $x_{1}, x_{2}, \ldots$ are collectively heavier than $y_{1}, y_{2}, \ldots$.

One collection of problems with van Inwagen style paraphrases of mereological talk are those concerning expressive power discussed in Uzquiano (2004). Uzquanio points out that sentences like the following cannot be dealt with by standard, van Inwagen style, translations which translate from the language of mereology into the language of plural logic with plural predicates.

(1) The chairs outnumber the tables.

The main issue here is that the most natural translation of this sentence in the language of mereology (extended by plural quantifiers) is simply, where $x x$ and $y y$ are plural variables, and $x \ll x x$ means " $x$ is one of the $x x$ ":

$$
\begin{aligned}
& \exists x x(\forall x(x \ll x x \leftrightarrow \operatorname{chair}(x)) \wedge \\
& \exists y y(\forall y(y \ll y y \leftrightarrow \text { table }(y)) \wedge \\
& \text { outnumber }(x x, y y))) .
\end{aligned}
$$

This sentence, though, requires plural quantification over tables and chairs, and if we were to naively apply the translation procedure advocated by van Inwagen then we would end up needing plural collections of plural collections of atoms of chairs/ tables respectively. This highlights the first of the two problems which Uzquiano poses for any attempt to 'do away' with talk of mereologically composite objects, these being (i) how are we to explain plural quantification over composite objects, and (ii) how are we to deal with plural predicates which are collectively satisfied by complex objects.

In the obvious extension of our language by plural quantifiers and predicates sentence (1) can be formalised as follows.

\footnotetext{
14 We have changed vanInwagen's notation for plural variables to what is now considered standard. Here plural variables are denoted as $x x, y y, z z$ etc, with the plural extension of a given language adding plural variables and quantifiers which bind them, as well as a new primitive predicate $<<$ which takes a singular term in its first position and a plural term in its second, with $x<<x x$ being read as " $x$ is among the $x x \mathrm{~s}$ ".
} 


$$
\begin{aligned}
& \exists x x\left(\forall x_{1} \cdots \forall x_{n} \cdots\left(\left(\bigwedge_{i<\omega}\left(x_{i} \ll x x\right)\right) \leftrightarrow \operatorname{chair}^{f}\left(x_{1}, \ldots, x_{n}, \ldots\right)\right) \wedge\right. \\
& \exists y y\left(\forall y_{1} \cdots \forall y_{n} \cdots\left(\left(\bigwedge_{i<\omega}\left(y_{i} \ll y y\right)\right) \leftrightarrow \operatorname{table}^{f}\left(y_{1}, \ldots, y_{n}, \ldots\right)\right) \wedge\right. \\
& \text { outnumber } \left.\left._{\text {chair }, \text { table }}(x x, y y)\right)\right)
\end{aligned}
$$

The main thing worth noting here is that we have had to relativize our 'outnumbering' predicate to a pair of sortal terms (which determine what kinds of collections are meant to be outnumbering what other kinds of collections). This points towards our general strategy for dealing with the second of Uzquino's problems. In general, the idea is that we can translate a collective predication $P(x x)$ where the $x x$ are of complex objects of kind $K$ in terms of the collective satisfaction of the plural predicate $P_{K}\left(x x^{\prime}\right)$ where the $x x^{\prime}$ are all the simple parts of members of $x x$. In order for a plurality of atoms $x x$ to satisfy a predicate $P_{K}$ what is required, amongst other things, is that the members of $x x$ can be partitioned up into collections of simples $s_{1}, \ldots, s_{n}, \ldots$ s.t. $K^{f}\left(s_{1}, \ldots, s_{n}, \ldots\right)$. Depending on the predicate $P$, other conditions will need to be met by the $x x \mathrm{~s}$. Sometimes there will be illuminating things which can be said about what those additional conditions may be. For example, for the

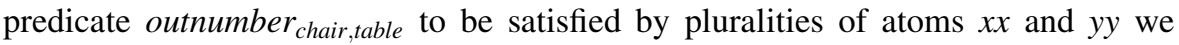
require:

- The $x x$ can be partitioned into collections of simples which satisfy chair $^{f}$

- The yy can be partitioned into collections of simples which satisfy table

- There is a function $f$ from members of $x x$ to sets $s$ of members of $y y$ s.t.

- for every $x \ll x x$, and enumeration $s_{1}, \ldots, s_{n}, \ldots$ of $f(x)$ we have table $^{f}\left(s_{1}, \ldots, s_{n}, \ldots\right)$

- for all $a_{1}, \ldots, a_{n}, \ldots$ in $x x$ if $\operatorname{chair}^{f}\left(a_{1}, \ldots, a_{n}, \ldots\right)$ then $f\left(a_{i}\right)=f\left(a_{j}\right)$

- There is no similar function from members of $y y$ to sets of members of $x x$ (with table $f^{f}$ and chair $^{f}$ swapped as imagined)

There will be situations, though, where we cannot provide illuminating further conditions (aside from partition conditions) which need to be met by a collection of simples to be satisfied by a flexible plural predicate. So, for example, consider the case of the predicate 'touching one another' in the following sentence.

(2) Some bricks are touching one another.

In this case there is nothing particularly illuminating we can say about what it takes for some atoms $x x$ to satisfy touching $_{\text {brick }}$ aside from that they must be able to be partitioned into collections which satisfy brick ${ }^{f}$. This is mostly because the kind of arrangement which atoms must stand in in order to be constitute some touching bricks isn't readily specifiable. 
A similar proposal is discussed in Uzquiano (2004, p. 447), according to which we translate predicates which are collectively satisfied by a plural argument, like 'touching one another' in "Some bricks are touching one another", in terms of predicates like 'brick-touching-one-another', where BRICK-TOUCHING-ONE-ANOTHER $(x x)$ is true iff the members of $x x$ can be partitioned up into a collection of sets $s s$, the members of each set being arranged brick-wise, such that the ss are touching one another. This approach is found wanting by Uzquiano because it treats sentences like (2) as being 'about' sets, it makes it too easy for (2) to be true, and according to it the translation of (2) doesn't entail the translation of "There are bricks". Our proposal, not involving sets in any manner, does not fall afoul of the first objection here, and on a plausible account of the behaviour of infinitary quantification the appropriate translation of (2) will entail the translation of "There are bricks." This leaves Uzquano's third criticism, which (even in the case he is considering) appears to depend on the atoms which make up the two halves of a brick being arranged brick-wise. If this is the case then, plausibly, the mereological sum of half a brick is a brick (these being the kinds of facts which predicates of arrangement are intended to track), in which case our translation will track what the defender of classical extensional mereology would say about such a case. This being our aim, we can dispense with this objection. ${ }^{15}$

In this section our approach has merely been to show that our strategy does not fall afoul of Uzquano's expressive power worries, and in the process to sketch how our approach could be extended to languages which also contained plural quantification.

\section{Innocence Gained}

The ontological commitments of a theory are the constraints it places upon world concerning what entities there are (cf. Rayo 2007). The worry of the person who thinks that mereology is not ontologically innocent is that it places additional constraints upon the world concerning what entities there are: forcing there to be not only cats, but also cat-fusions, not just cats and mice, but also cat-mouse-fusions like Tom-and-Jerry. What the above translation shows, though, is that all atomistic classical mereology commits us too (in the thick sense) is the existence of mereological atoms. Speaking more precisely, what we take the aforementioned translation result to show is that we can characterise the portions of reality which contain mereologically complex objects solely in terms of their parts and the various flexible relations which they stand in-so that ultimately, in the absence of gunk, the world can be characterised completely in terms of mereological atoms and the various flexible relations which they stand in. ${ }^{16}$ Moreover, our translation suggests a

\footnotetext{
15 This may simply suggest to the reader that this is (perhaps yet another way) in which Classical Extensional Mereology gets things wrong. Our aim here, though, it not to defend classical extensional mereology against all attacks, rather it is merely to explain how it might be considered to be ontologically innocent.

16 This may not be the most perspicuous way to represent the way the world is in fundamental terms, though, especially if one holds, as Lewis did, that the fundamental relations are those that would appear in
} 
way of understanding Lewis' claim that mereological relations are like identity. On the above account 'parthood' is like identity in the sense that every statement of parthood in a mereological language can be treated as a statement in the flexibleinfinitary language as a complex identity statement between the parts of the objects involved. Given that all of the mereological relations can be defined in terms of parthood, it follows that (in a perhaps somewhat extended sense) mereological relations are like identity. Furthermore, and more importantly, the above translations also show how facts about mereological fusions can be explained in terms of facts about the things fused.

\section{Whither Nihilism?}

Our formal results require that we are considering atomistic classical extensional mereology. This may lead one to, quite reasonably, believe that we are committed to there being mereological atoms, at which point our view looks quite a lot like mereological nihilism - the translation above illustrating how to paraphrase away talk of mereologically complex objects. One reason for this appearance could be the reaction that, in the face of mereological atomism, the dispute between nihilists and universalists appears to look somewhat insubstantial (Putnam 1987, p. 70). These appearances are, I think, deceptive, especially in the current case.

Falling into the above temptation requires one to understand the above results as showing that, if our assumption No Gunk is true, then the nihilist can say everything which the mereological universalist can (our translation indicating how this can be done). After all, as I explained above, the present approach does look quite a lot like van Inwagen's paraphrasing away of talk of composite objects, with his plural quantification being cashed out in terms of infinitary quantification, and his plural predicates replaced with flexible predicates and relations between mereological atoms. ${ }^{17}$

This comparison only gets off the ground if the atoms in our mereological model correspond to objects which have no proper parts (henceforth atoms), and there is no need for this to be the case. There are all sorts of reasons to be interested in the objects which we can construct out of a given collection of objects. As is pointed out

Footnote 16 continued

a completed fundamental physics. In this case we would expect the various flexible predicates and relations to be defined (in a not necessarily uniform manner) in terms of those relations. This would make the relationship between a description in terms of mereological atoms and flexible relations and one in terms of mereological fusions and 'standard' relations much like the relationship between a description of a system given in biological terms, and one given in microbiological or chemical terms.

17 Once we draw the distinction between thick and thin commitments the dispute between nihilists and universalists becomes slightly more complicated, depending on the manner in which the nihilist is denying a commitment to mereologically complex objects. For the nihilist position to truly be incompatible with the universalist position being argued for here they would have to claim that we are not thinly committed to mereologically complex objects, and thus object either to the claim that both $L_{m}$ and $L_{\omega_{1} \omega_{1}}^{f}$ are equally ontologically revealing, or our characterisation of thick and thin commitments given above. What this definitely does seem to illustrate is that, even if our assumption No Gunk is true the dispute between nihilists and universalists is still a substantial one. 
in Fine (1991), there is no need for the basic objects in our ontology to be the ones which cannot be generated using our various 'constructors' from other objects- the example that Fine uses to make this point being particularly relevant to our purposes. $^{18}$

A more significant example concerns a universe of indefinitely divisible matter of certain basic kinds. Any uniform piece of matter will then be the aggregate of smaller pieces of matter. But all the same, it is reasonable to suppose that the uniform pieces of matter are all basic. They do not earn their admission into the ontology as constructs, even though any one of them might have been regarded as a construct (Fine 1991, p. 266).

So one way we can conceive of the atoms of our mereological model is as some collection of objects which we've singled out as basic, with our mereology allowing us to talk about those objects and their fusions. Thinking of our atoms in this way, what our results show is that anything which we can say about fusions of our basic objects we can say directly about the basic objects themselves in a sufficiently expressive languages without committing ourselves to the existence of any further objects.

If the world is gunky then there is a very natural place where we might look for such basic objects. Instead of thinking of our atomistic mereological theory as modelling the full mereological structure of the world, thus making its atoms just be atoms, we could instead think of it as modelling the fundamental explanatory structure of the world. Here the thought is that the mereological atoms in our mereological theory (and thus the entities in the domain of quantification for our $L_{\omega_{1} \omega_{1}}^{f}$-models) are the smallest entities needed in order to tell a complete story about Reality. Now these entities may be gunky, so long as their parts play no (or at least no substantive) role in explanation. Schaffer, talking about the inference from the postulation of particles in a completed microphysics, to the conclusion that the particles are mereological atoms says the following:

That the particles are postulated by a complete microphysics shows only that one can tell a complete causal story with particles as protagonists. But one can tell a complete causal story with divisible protagonists, provided that the divisions are boring, in that the characteristic properties of all the parts supervene on the characteristic properties of their wholes (Schaffer 2003, p. 505).

Adopting this idea, then, the results above can be used to show that mereology is ontologically innocent, even in the presence of atomless gunk, so long as there is some fundamental supervenience base, which consists of objects which are either atoms or are boring in the sense described above.

\footnotetext{
${ }^{18}$ Fine (1991) divides ontologies into their basic elements, constructors, and their elements. So, in the case of (pure) set-theory the basic elements will be the empty set, the constructor the set-building operation $\{\cdot\}$, and the elements all of the pure sets. In the mereological case things are not so clear, though - as we highlight here. Obviously our constructor will be the operation of mereological fusion, but what the basic objects are is somewhat less obvious.
} 
At this point we should qualify the statement we gave in Sect. 2 of what is required for mereology to be ontologically innocent. Under the assumption of No Gunk what is required for the ontological innocence of mereology according to INNOCENCE is that talk of mereologically complex objects can be cashed out (via a translation) in terms of talk about mereological atoms. It could well be, though, that the fundamental supervenience base in fact involves composite objects of some kind. In this case, then, there is a sense in which we can show that mereology is ontologically innocent in a more extended sense. In this more extended sense, what is required is that mereology brings with is no additional ontological commitments-i.e. that the atoms of our mereological models are those which we are antecedently committed to. Thinking of ontological innocence in this more extended sense appears to be the best way of making sense of the talk in Lewis (1991, p. 82) of mereology being innocent because it brings with it no further ontological commitments, that it "commits us only to things ... [that] we were committed to before". In the case where No Gunk is false, but we have a fundamental supervenience base of boring objects, we can claim an (admittedly qualified) kind of ontological innocence for mereology. If we are constructing a total metaphysical theory we are antecedently committed to the objects which appear in the fundamental supervenience base (be they atomic or composite), as they and the properties they stand in underwrite all truths. The addition of mereological primitives adds no further commitment over and above this. So in this sense, then, mereology would not be innocent regarding the existence of mereologically complex objects, but rather it would be innocent concerning the existence of mereologically complex objects which are not part of the fundamental supervenience base.

\section{Revisiting Our Assumptions}

What we have shown is that there is good reason to think that mereology is ontologically innocent, the seemingly extra (thick) ontological commitments of mereology to fusions over and above the things fused being simply a result of mereology encoding underlying commitments to the things fused in a manner which we can avoid by using more extensive expressive devices. Our discussion above was still relative to the number of assumptions we made in Sect. 2. In the previous section we have already discussed how we can at least weaken, if not lift entirely, the assumption of No Gunk. We will now return to our other assumptions, where the situation is largely much simpler.

Lifting the assumption Worldbound requires us to make a stand on a number of issues largely orthogonal to the present discussion. One formal way of doing this which would retain some degree of neutrality is to work in terms of an extensional theory of possible worlds such as Lewis's (1968) counterpart theory. Here the idea would be to consider an extension of the first-order mereological theory so that we also have all the distinguished predicates of counterpart theory (most importantly the counterpart relation), and so in our infinitary flexible first-order language we 
would end up with a corresponding flexible counterpart relation between the atoms of individuals in different worlds.

The restriction on the size of our mereological models (e.g. considering only models which have at most denumerably many mereological atoms) was purely one of convenience, allowing us to deal with an extension the more familiar looking infinitary language $L_{\omega_{1} \omega_{1}}$. All we would need to do in order to deal with larger models is to alter the infinitary part of our language appropriately. One option would be to simply work in terms of the language $L_{\infty \infty}$ which allows blocks of quantifiers and infinite conjunctions/disjunctions of arbitrary size.

There is one other assumption which we have not made explicitly above, but which it is worth keeping in mind here. Our original statement of INNOCENCE concerned theories such as classical extensional mereology, but our formal results (of necessity) concern a particular regimentation of that theory. In order for our formal results to show that mereology is indeed ontologically innocent according to INNOCENCE above we are implicitly assuming that this presentation is faithful in the relevant respects to classical extensional mereology. In particular one commitment which we have incurred (which have have aimed to partially address in Sect. 6) is that formal results like those we appeal to will hold in regimentations of classical extensional mereology with different expressive resources. It is classical extensional mereology itself which we claim is ontologically innocent, not merely any particular formal regimentation of it. ${ }^{19}$

\section{Conclusion}

Lewis was right to think that there was an intimate relation between mereological primitives and identity - in describing the ultimate furniture of reality mereological talk ends up only placing requirements on reality concerning the identity and distinctness of the objects: be they mereological atoms, various objects which we are taking as givens in our ontological theorising, or perhaps the elements of the fundamental supervenience base. I suspect my argument for this conclusion is perhaps not one Lewis himself would have favoured, but I think it lends stronger support to the notion than his argument by analogy. The cats and the cat fusions really are the same portion of reality, just as Lewis said.

Acknowledgments I would like to thank Lloyd Humberstone, Greg Restall, Alastair Wilson and a number of referees for this journal for their helpful comments.

Open Access This article is distributed under the terms of the Creative Commons Attribution 4.0 International License (http://creativecommons.org/licenses/by/4.0/), which permits unrestricted use, distribution, and reproduction in any medium, provided you give appropriate credit to the original author(s) and the source, provide a link to the Creative Commons license, and indicate if changes were made.

\footnotetext{
${ }^{19}$ I'd like to thank an anonymous referee for raising this particular point.
} 


\section{Technical Appendix}

In this appendix we will go over the technical results which are at the heart of this paper in more detail.

\section{Languages}

\section{The Mereological Language}

Let $L_{m}$ be a first-order language with denumerable supply of predicates $P_{1}^{n}, \ldots, P_{n}^{n}, \ldots$ of each arity $n$, denumerably many variables $v_{1}, \ldots, v_{n}, \ldots$, the universal quantifier ' $\forall$ ', identity ' $=$ ', the boolean connectives ' $\wedge$ ' and ' $\neg$ ', and a distinguished binary relation ' $\square$ ' for parthood. The existential quantifier and other boolean connectives are defined as usual.

Models for $L_{m}$ are structures $\langle D, \sqsubseteq, I\rangle$ where $\langle D, \sqsubseteq\rangle$ is isomorphic to the structure $\langle S, \subseteq\rangle$ where $S=\wp(X) \backslash \emptyset$ and $X$ is the set of minimal elements of $D$ relative to $\sqsubseteq$. Truth of a formula $\varphi$ of $L_{m}$ in a model $\mathcal{M}=\langle D, \sqsubseteq, I\rangle$ on an assignment of elements of $D$ to free-variables $\mu$ (written " $\mathcal{M} \models \varphi[\mu]$ ") is defined inductively as follows.

$$
\begin{aligned}
\mathcal{M} \models P v_{1} \ldots v_{n}[\mu] & \Longleftrightarrow \\
\mathcal{M} \models v_{i}=v_{j}[\mu] & \Longleftrightarrow \\
\mathcal{M} \models v_{i} \sqsubseteq v_{j}[\mu] & \Longleftrightarrow \\
\mathcal{M} \models \neg \varphi[\mu] & \Longleftrightarrow \\
\mathcal{M} \models \varphi \wedge \psi[\mu] & \Longleftrightarrow \\
\mathcal{M} \models \forall v_{i} \varphi[\mu] & \Longleftrightarrow
\end{aligned}
$$

$$
\begin{gathered}
\left\langle\mu\left(v_{1}\right), \ldots, \mu\left(v_{n}\right)\right\rangle \in I(P) . \\
\mu\left(v_{i}\right)=\mu\left(v_{j}\right) . \\
\mu\left(v_{i}\right) \sqsubseteq \mu\left(v_{j}\right) . \\
\mathcal{M} \forall \varphi[\mu] . \\
\mathcal{M} \models \varphi[\mu] \text { and } \mathcal{M} \models \psi[\mu] . \\
\mathcal{M} \models \varphi\left[\mu^{\prime}\right] \text { for all } \mu^{\prime}
\end{gathered}
$$

which differ from $\mu$ at most on $v_{i}$.

\section{The Flexible Infinitary Language}

The language $L_{\omega_{1} \omega_{1}}^{f}$ is a first-order language with a denumerable supply of flexible predicates $P^{f^{n}}, \ldots, P_{n}{ }_{n}^{n}, \ldots$ of each arity $n$, an uncountable supply of variables $x_{i}$ $\left(i \leq \omega_{1}\right)$, the universal quantifier ' $\forall$ ', identity ' $=$ ', conjunction ' $\wedge$ ', disjunction ' $\vee$ ' and negation ' $\neg$ '. Recall that a flexible predicate forms formulas by putting sequences of terms in its argument places the size of which are bounded by its place-limitation.

A place-limitation $\ell$ is a pair $[i ; \alpha]$ where $i$ is a natural number, and $\alpha$ is an ordinal, with $i$ being the lower limit of $\ell$ and $\alpha$ being the upper limit of $\ell$, which we will denote respectively by $l l(\ell)$ and $u l(\ell)$. The type of a flexible predicate $F$ of degree $n$ is then a sequence $\left\langle\ell_{1}, \ldots, \ell_{n}\right\rangle$ of place-limitations.

The formation rules for $L_{\omega_{1} \omega_{1}}^{f}$ are as follows. 
- If $P^{f}$ is an $n$-ary flexible predicate with place limitation $\left\langle\ell_{1}, \ldots, \ell_{n}\right\rangle$, and $x_{1}^{1}, \ldots, x_{i_{1}}^{1}, \ldots, x_{1}^{n}, \ldots, x_{i_{n}}^{n}$ are variables where each $i_{j}$ is an ordinal less that $u l\left(\ell_{j}\right)$ then $P^{f}\left(x_{1}^{1}, \ldots, x_{i_{1}}^{1} ; \ldots ; x_{1}^{n}, \ldots, x_{i_{n}}^{n}\right)$ is a formula.

- If $x_{i}$ and $x_{j}$ are variables then $x_{i}=x_{j}$ is a formula.

- If $\varphi$ and $\psi$ are formulas then so are $\varphi \wedge \psi, \varphi \vee \psi$ and $\neg \varphi$.

- If $\varphi_{1}, \ldots, \varphi_{n}, \ldots$ is a sequence of formulas of length $<\omega_{1}$ then $\varphi_{1} \vee \cdots \vee \varphi_{n} \vee$ $\cdots$ and $\varphi_{1} \wedge \cdots \wedge \varphi_{n} \wedge \cdots$ are formulas.

- If $\varphi$ is a formula and $x_{i}$ a variable then $\forall x_{i} \varphi$ is a formula.

- If $x_{1}, \ldots, x_{n}, \ldots$ is a sequence of variables of length $<\omega_{1}$ none of which occur bound in $\varphi$ then $\forall x_{1} \cdots \forall x_{n} \cdots \varphi$ and $\exists x_{1} \cdots \exists x_{n} \cdots \varphi$ are formulas.

A model for $L_{\omega_{1} \omega_{1}}^{f}$ is a structure $\langle D, I\rangle$ where $D$ is a non-empty set, and $I$ a first-order interpretation function which meets to following constraint: for all flexible predicates $F$ of type $\left\langle\ell_{1}, \ldots, \ell_{n}\right\rangle$ the interpretation $I$ of our models for $L_{\omega_{1} \omega_{1}}^{f}$ assigns a set of $n$-tuples $\left\langle s_{1}, \ldots, s_{n}\right\rangle$ s.t. for all $i$ where $1 \leq i \leq n$ :

(i) each $s_{i}$ is a sequence of elements of $D$,

(ii) $\quad l l\left(\ell_{i}\right) \leq$ length $\left(s_{i}\right) \leq u l\left(\ell_{i}\right)$.

Truth of a formula $\varphi$ of $L_{\omega_{1} \omega_{1}}^{f}$ in a model $\mathcal{M}=\langle D, I\rangle$ on an assignment of elements of $D$ to free variables $\mu$ (written " $\mathcal{M} \Vdash \varphi[\mu]$ ") is defined inductively as follows, where $\overrightarrow{\mathbf{x}}_{i}$ is an abbreviation for $x_{1}, \ldots, x_{i}$.

$$
\begin{array}{ccc}
\mathcal{M} \Vdash P^{f}\left(\mathbf{x}_{\mathbf{i}_{1}}^{\overrightarrow{1}} ; \ldots ; \mathbf{x}_{\mathbf{i}_{\mathbf{n}}}^{\overrightarrow{\mathbf{n}}}\right)[\mu] & \Longleftrightarrow & \left\langle\left\langle\mu\left(\mathbf{x}_{\mathbf{i}_{1}}^{\overrightarrow{\mathbf{n}}}\right)\right\rangle, \ldots,\left\langle\mu\left(\mathbf{x}_{\mathbf{i}_{\mathbf{n}}}^{\overrightarrow{\mathbf{n}}}\right)\right\rangle\right\rangle \in I\left(P^{f}\right) . \\
\mathcal{M} \Vdash x_{i}=x_{j}[\mu] & \Longleftrightarrow & \mu\left(x_{i}\right)=\mu\left(x_{j}\right) . \\
\mathcal{M} \Vdash \neg \varphi[\mu] & \Longleftrightarrow & \mathcal{M} \nVdash \varphi[\mu] . \\
\mathcal{M} \Vdash \varphi \wedge \psi[\mu] & \Longleftrightarrow & \mathcal{M} \Vdash \varphi[\mu] \text { and } \mathcal{M} \Vdash \psi[\mu] . \\
\mathcal{M} \Vdash \forall x \varphi[\mu] & \Longleftrightarrow & \mathcal{M} \Vdash \varphi\left[\mu^{\prime}\right] \text { for all } \mu^{\prime} \\
\mathcal{M} \Vdash \forall x_{1} \cdots \forall x_{n} \cdots \varphi[\mu] & \Longleftrightarrow & \text { for all assignments } \mu^{\prime} \text { differing at most } \\
\mathcal{M} \Vdash \varphi_{1} \vee \cdots \vee \varphi_{n} \vee \cdots[\mu] & \Longleftrightarrow & \mathcal{M} \Vdash \varphi_{1}[\mu] \text { or } \cdots \mathcal{M} \Vdash \varphi \varphi_{n}[\mu] \text { or } \cdots
\end{array}
$$

\section{Translation Proofs}

For our translation we assume that there is a injective function $g$ between $n$-ary predicates of $L_{m}$ and $n$-place flexible predicates of $L_{\omega_{1} \omega_{1}}^{f}$ whose place limitations are $\langle[1 ; \omega], \ldots,[1 ; \omega]\rangle$. We note this by writing in our translation $R^{f}$ to pick out the predicate $g(R)$ where $R$ is a predicate from $L_{m}$ to save clutter. 


$$
\begin{aligned}
\tau\left(R v_{1} \ldots v_{n}\right)= & R^{f}\left(x_{1}^{v_{1}}, x_{2}^{v_{1}}, \ldots ; \ldots ; x_{1}^{v_{n}}, x_{2}^{v_{n}}, \ldots\right) . \\
\tau(\forall v \varphi)= & \forall x_{1}^{v} \forall x_{2}^{v} \cdots \tau(\varphi) . \\
\tau(v \sqsubseteq u)= & \forall x\left(\left(x=x_{1}^{v} \vee x=x_{2}^{v} \vee \cdots\right) \rightarrow\right. \\
& \left.\exists y\left(\left(y=x_{1}^{u} \vee y=x_{2}^{u} \vee \cdots\right) \wedge x=y\right)\right) . \\
\tau(v=u)= & \tau(u \sqsubseteq v) \wedge \tau(v \sqsubseteq u) .
\end{aligned}
$$

In order to determine whether a given object in the models for our mereological language is an atom we will find it useful to use the unary predicate Atom $(x)$, which is defined as

$$
\operatorname{Atom}(x)={ }_{D e f} \forall z(z \sqsubseteq x \rightarrow z=x) .
$$

That is to say, an object $x$ is a mereological atom if its only part is itself. Given a model $\langle D, \sqsubseteq, I\rangle$ an an $a \in D$ let:

$$
\text { Atomic-Parts }(a)=\left\{a_{i} \in D \mid \operatorname{Atom}\left(a_{i}\right) \& a_{i} \sqsubseteq a\right\} .
$$

Definition 1 Given a model for atomistic classical mereology $\mathcal{M}=\langle D, \sqsubseteq, I\rangle$ let $\mathcal{M}^{-}=\left\langle D^{-}, I^{-}\right\rangle$be defined as follows.

- $D^{-}:=\{a \in D \mid a \in I($ Atom $)\}$.

- $I^{-}(R):=\left\{\left\langle A P\left(a_{1}\right), \ldots, A P\left(a_{n}\right)\right\rangle:\left\langle a_{1}, \ldots, a_{n}\right\rangle \in I(R)\right.$ where $A P\left(a_{i}\right)$ is an enumeration of Atomic-Parts $\left(a_{i}\right)$.

This puts us in the position of being able to state our main result.

Theorem 2 For all models of atomistic classical mereology $\mathcal{M}=\langle D, \sqsubseteq, I\rangle$ with at most denumerably many atoms there is a unique model $\mathcal{M}^{-}=\left\langle D^{-}, I^{-}\right\rangle$s.t. for all sentences $\varphi$ in the mereological language we have the following:

$$
\mathcal{M} \models \varphi \text { if and only if } \mathcal{M}^{-} \Vdash \tau(\varphi) .
$$

In order to prove our main result we will first need to prove an auxiliary proposition.

Definition 3 Given a model $\mathcal{M}=\langle D, \sqsubseteq, I\rangle$ and assignment $\mu$ for $L_{m}$ and a model $\mathcal{M}^{-}=\left\langle D^{-}, I^{-}\right\rangle$and an $L_{\omega_{1} \omega_{1}}^{f}$ assignment $\mu^{\prime}$ say that $\mu$ and $\mu^{\prime}$ atom-agree on $\varphi$ for $\varphi \in L_{m}$ whenever, for all free-variables $v_{i}$ in $\varphi$ we have

$$
\mu^{\prime}\left(x_{j}^{v_{i}}\right)=a_{j}
$$

where $a_{j}$ is the $j$-th member of an enumeration of $\operatorname{Atomic-Parts}\left(\mu\left(v_{i}\right)\right)$ if $j \leq \mid$ Atomic-Parts $\left(\mu\left(v_{i}\right)\right) \mid$ and is equal to the first member of the enumeration otherwise. 
What this means is that whenever we have two variable assignments $\mu$ and $\mu^{\prime}$ which atom agree on an $L_{m}$-formula $\varphi$ then we know that if $v$ is free in $\varphi$ then the variables $x_{1}^{v}, \ldots, x_{i}^{v}, \ldots$ will be assigned on $\mu^{\prime}$ all and only the atomic parts of the object $\mu(v)$.

Proposition 4 For all $\varphi \in L_{m}$, all models $\mathcal{M}=\langle D, \sqsubseteq, I\rangle$ with at most denumerably many atoms and all assignments $\mu$ :

$$
\mathcal{M} \models \varphi[\mu] \Longleftrightarrow \mathcal{M}^{-} \Vdash \tau(\varphi)\left[\mu^{\prime}\right]
$$

where $\mu$ and $\mu^{\prime}$ atom-agree.

Proof By induction on the complexity of $\varphi$. We treat the most interesting cases here, that in the basis case where $\varphi=v \sqsubseteq u$ and that in the inductive step where $\varphi=\forall v \psi$ for some $\psi$ of lesser complexity that $\varphi$. The other cases are either routine, or in the case of identity a trivial modification of the $\sqsubseteq$ case.

(巨): Suppose that $\mathcal{M} \models v \sqsubseteq u[\mu]$. This is the case iff $\mu(v) \sqsubseteq \mu(u)$ which is the case iff Atomic-Parts $(\mu(v)) \subseteq$ Atomic-Parts $(\mu(u))$. For any $\mu^{\prime}$ which atom-agrees with $\mu$ on $\varphi$ this means that

$$
\begin{aligned}
&\left\{\mu^{\prime}\left(x_{i}^{v}\right) \mid \mu^{\prime}\left(x_{i}^{v}\right) \in \text { Atomic-Parts }(\mu(\mathrm{v}))\right\} \subseteq \\
&\left\{\mu^{\prime}\left(x_{i}^{u}\right) \mid \mu^{\prime}\left(x_{i}^{u}\right) \in \text { Atomic-Parts }(\mu(u))\right\} .
\end{aligned}
$$

This is the case iff

$$
\mathcal{M}^{-} \Vdash \forall x\left(\left(x=x_{1}^{v} \vee x=x_{2}^{v} \vee \cdots\right) \rightarrow \exists y\left(\left(y=x_{1}^{u} \vee y=x_{2}^{u} \vee \cdots\right) \wedge x=y\right)\right) \cdot\left[\mu^{\prime}\right],
$$

which is equivalent to $\mathcal{M}^{-} \Vdash \tau_{s}(v \sqsubseteq u)\left[\mu^{\prime}\right]$ as desired.

$(\forall)$ : suppose that $\mathcal{M} \models \forall v \psi[\mu]$. Then for all $\mu^{*}$ s.t. $\mu^{*}$ and $\mu$ agree on all variables but $v$ we have $\mathcal{M} \models \psi\left[\mu^{*}\right]$. So by the induction hypothesis we have $\mathcal{M} \models \tau_{s}(\psi)\left[\mu^{*^{\prime}}\right]$ where $\mu^{*}$ and $\mu^{*^{\prime}}$ atom-agree on $\psi$. By inspection of the translation, and the construction of $I^{-}$it is easy to see that the particular enumeration used to show that $\mu^{*}$ and $\mu^{*^{\prime}}$ atom-agree on $\psi$ does not effect the satisfiability of formulas of the form $\tau(\psi)$, and thus in fact every enumeration $e$ of the atomic parts of $\mu^{*}(v)$ will give us an assignment $\mu_{e}^{*^{\prime}}$ which atom-agrees with $\mu^{*}$ on $\psi$. Thus it follows that $\mathcal{M}^{-} \Vdash \forall x_{1}^{v} \forall x_{2}^{v} \cdots \tau(\psi)\left[\mu^{\prime}\right]$ as required.

Suppose now that $\mathcal{M}^{-} \Vdash \forall x_{1}^{v} \forall x_{2}^{\nu} \cdots \tau(\psi)\left[\mu^{\prime}\right]$. Then for all assignments $\mu^{\prime \prime}$ which are $x_{1}^{v}, x_{2}^{v}, \ldots$-variants of $\mu^{\prime}$ we have that $\mathcal{M}^{-} \Vdash \tau(\psi)\left[\mu^{\prime \prime}\right]$. So by the induction hypothesis we have $\mathcal{M} \models \psi\left[\mu^{\prime \prime}\right]$ where $\mu^{\prime \prime *}$ atom-agrees with $\mu^{\prime \prime}$ on $\psi$. But this means $\mathcal{M}$ satisfies $\psi$ for all $v$-variant assignments of the assignment $\mu$ which atomagrees with $\mu^{\prime}$-as whenever $S$ is the smallest set $S \subseteq D^{-}$s.t. for all $i \mu^{\prime \prime}\left(x_{i}^{v}\right) \in S$ we have that $\mu^{\prime *}(v)=a$ where $a$ is the object whose atomic parts are all and only those in $S$. Thus we can conclude that $\mathcal{M} \models \forall v \psi[\mu]$ as desired.

It is easy to see that our main theorem follows from the above proposition, being the special case where $\varphi$ is a sentence. 


\section{References}

Baxter, D. L. M. (1988). Identity in the loose and popular sense. Mind, 97, 575-582.

Bell, J. L. (2012). Infinitary logic. http://plato.stanford.edu/entries/logic-infinitary/.

Comesaña, J. (2008). Could there be exactly two things? Synthese, 162, 31-35.

Cotnoir, A. J., \& Baxter, D. L. M. (2014). Composition as Identity. Oxford: Oxford University Press.

Fine, K. (1977). Postscript: Prior on the construction of possible worlds and instants. In A. N. Prior \& K. Fine (Eds.), Worlds. Duckworth: Times and Selves.

Fine, K. (1991). The study of ontology. Noûs, 25, 263-294.

Fine, K. (2010). Towards a theory of part. Journal of Philosophy, 107(11), 559-589.

Gödel, K. (1944). Russell's mathematical logic. In P. A. Schilpp (Ed.), The philosophy of Bertrand Russell (pp. 123-153). Evanston and Chicago: Northwestern University.

Hodes, H. (1990). Ontological commitment: Thick and thin. In G. Boolos (Ed.), Meaning and method: Essays in honor of Hilary Putnam (pp. 347-407). Cambridge: Cambridge University Press.

Karmo, T. (1985). Are singular terms needed for describing the world? Australasian Journal of Philosophy, 63, 419-427.

Lewis, D. (1968). Counterpart theory and quantified modal logic. Journal of Philosophy, 65(5), 113-126.

Lewis, D. (1986). On the plurality of worlds. Oxford: Blackwell.

Lewis, D. (1991). Parts of classes. Oxford: Basil Blackwell.

Linnebo, Ø. (2012). Metaontological minimalism. Philosophy Compass, 7, 139-151.

Morton, A. (1975). Complex individuals and multigrade relations. Nô̂s, 9, 309-318.

Putnam, H. (1987). Truth and convention: On Davidson's refutation of conceptual relativism. Dialectica, $41,69-77$.

Quine, W. V. (1947). On universals. The Journal of Symbolic Logic, 12, 74-84.

Rayo, A. (2007). Ontological commitment. Philosophy Compass, 2, 428-444.

Schaffer, J. (2003). Is there a fundamental level? Nô̂s, 37(3), 498-517.

Sider, T. (1993). Van Inwagen and the possibility of gunk. Analysis, 53, 285-289.

Sider, T. (2007). Parthood. Philosophical Review, 116, 51-91.

Simons, P. M. (1987). Parts: A study in ontology. Oxford: Oxford University Press.

Taylor, B., \& Hazen, A. P. (1992). Flexibly structured predication. Logique et Analyse, 139-140, 375-393.

Uzquiano, G. (2004). Plurals and simples. The Monist, 87, 429-451.

van Inwagen, P. (1990). Material beings. Ithaca and London: Cornell University Press.

Williams, J. R. G. (2012). Requirements on reality. In C. Fabrice \& B. Schnieder (Eds.), Metaphysical grounding: Understanding the structure of reality (pp. 165-185). Cambridge: cambridge University Press.

Yi, B.-U. (1999). Is mereology ontologically innocent? Philosophical Studies, 93, 141-160. 